\title{
TECNOLOGIA ASSISTIVA COMO MEIO FACILITADOR PARA INCLUSÃO DE PESSOAS COM DEFICIÊNCIA NO AMBIENTE ESCOLAR: ANÁLISE DE UM RECURSO PEDAGÓGICO
}

Ana Mayra Samuel da Silva ${ }^{1}$, José Eduardo de Oliveira Evangelista Lanuti², Manoel Osmar Seabra Junior ${ }^{1}$

'Universidade Estadual Paulista - UNESP, Programa de Pós-Graduação em Educação, Presidente Prudente, SP. ${ }^{2}$ Universidade Estadual de Campinas - UNICAMP, Programa de Pós-Graduação em Educação, Campinas, SP. E-mail: dulanuti@gmail.com, ana.mayra.ss@gmail.com, seabrajr.unesp@gmail.com.

Órgão de fomento: FAPESP

\section{RESUMO}

Este artigo teve por objetivo descrever e analisar de forma colaborativa às adaptações realizadas em um recurso pedagógico para ser constituído como uma Tecnologia Assistiva. Estes procedimentos ocorreram junto à disciplina intitulada "Metodologias em Educação Especial com ênfase na Análise de Recursos de Tecnologia Assistiva em Ambientes Inclusivos", ministrada no Programa de Pós-Graduação em Educação da Universidade Estadual Paulista "Júlio de Mesquita Filho", campus de Presidente Prudente/SP, neste corrente ano. A partir dos estudos realizados na disciplina foram utilizados critérios para elaboração e análise de recursos de Tecnologia Assistiva mediante apontamentos da literatura, os quais proporcionaram as adequações iniciais, seguida por readequações a partir de discussões em grupo, de forma colaborativa, por diferentes profissionais. Para análise metodológica neste estudo foram descritas as etapas de confecção, análise e readequação do recurso à ser constituído como uma Tecnologia Assistiva.

Palavras-chave: Educação Especial. Inclusão. Tecnologia Assistiva. Recurso Pedagógico. Funcionalidade.

\section{ASSISTIVE TECHNOLOGY AS MEDIA TRAINER FOR INCLUSION OF PERSONS WITH DISABILITIES IN SCHOOL ENVIRONMENT: ANALYSIS OF A EDUCATIONAL RESOURCE}

\begin{abstract}
This article aims to describe and analyze collaboratively to adjustments made in an educational resource to be constituted as an Assistive Technology. These procedures took place with the discipline entitled "Methods in Special Education with emphasis on Assistive Technology Resource Analysis in Inclusive Environments", taught in the Graduate Education of the State University Program Paulista "Julio de Mesquita Filho", Presidente Prudente campus / SP, this year. From the studies in the discipline were the criteria used for the preparation and analysis of Assistive Technology resources through literature notes, which provided the initial adjustments, followed by Readjustments from group discussions, collaboratively, by different professionals. For methodological analysis in this study were described steps of preparation, analysis and readjusting resource to be constituted as an Assistive Technology.

Keywords: Special education. Inclusion. Assistive Technology. Educational resource. Functionality.
\end{abstract}




\section{INTRODUÇÃO}

A necessidade de oferecer um ensino de qualidade para todos os estudantes, garantindo as mesmas oportunidades de participação e aprendizagem, a partir das possibilidades e necessidades individuais, exige um novo olhar para os processos de ensino e de aprendizagem.

Para inclusão, seja no meio educacional ou social, se faz necessária a revisão de métodos, estratégias, recursos e materiais adequados ou mesmo adaptados, neste caso, no meio escolar, de modo que o ensino seja baseado nas especificidades de cada indivíduo.

De acordo com a Política Nacional de Educação Especial na Perspectiva da Educação Inclusiva, documento elaborado pela Secretaria de Educação Continuada, Alfabetização, Diversidade e Inclusão (SECADI), e do MEC, em 2008, para que os Estudantes Público-Alvo da Educação Especial (EPAEE) tenham garantidas as mesmas oportunidades que os demais, é preciso, a partir de suas necessidades construir recursos propícios para suas necessidades. Os EPAEE segundo a Política Nacional de Educação Especial na Perspectiva da Educação Inclusiva (2008, p.15) são estudantes com deficiência (Auditiva, Física, Intelectual e Visual), transtornos globais de desenvolvimento e altas habilidades/superdotação.

A tecnologia, entendida como o conjunto de técnicas que facilitam à resolução de problemas diários, passa a ser indispensável, quando se almeja construir práticas inclusivas, pois torna possível a participação daqueles que possuem determinadas limitações. Entretanto, é necessário compreender que tipos de técnicas são essas que facilitam a inclusão dos estudantes, qual sua definição e de que forma podem ser utilizadas.

Baseado nestas premissas os autores aqui intitulados participaram da disciplina intitulada "Metodologias em Educação Especial com ênfase na Análise de Recursos de Tecnologia Assistiva em Ambientes Inclusivos", ministrada pelo Profo Dro Manoel Osmar Seabra Junior, oferecida no Programa de Pós-Graduação em Educação da Universidade Estadual Paulista "Júlio de Mesquita Filho", campus de Presidente Prudente/SP.

Durante a disciplina desenvolveu-se um trabalho colaborativo, em que cada grupo de participantes construiu um recurso pedagógico e, no momento de cada uma das apresentações os demais membros se colocavam no papel de juízes, de modo que analisavam os outros recursos, que foram construídos pelos demais participantes. Esta atividade teve por intuito analisar se o recurso pedagógico construído poderia ser considerado um recurso de Tecnologia Assistiva (TA), e, apontar possíveis mudanças e contribuições para adaptação do mesmo, considerando critérios da literatura.

Nesse sentido, o objetivo geral deste artigo foi descrever e analisar de forma colaborativa às adaptações realizadas em um recurso pedagógico para ser constituído como uma Tecnologia Assistiva.

\section{METODOLOGIA}

A partir dos estudos realizados na disciplina supracitada, acerca da definição de TA, para esta pesquisa foi adotada a pesquisa exploratória do tipo descritiva analítica, que, de acordo com Cook (1995) é baseada em critérios pré-estabelecidos e aplicados, com avaliação criteriosa e reprodutível, resultando em uma síntese.

Participaram, colaborativamente, da disciplina intitulada "Metodologias em Educação Especial, com ênfase na Análise de Recursos de Tecnologia Assistiva em Ambientes Inclusivos", ministrada pelo Profo Dro Manoel Osmar Seabra Junior, os alunos pós-graduandos de diferentes áreas, a saber: Matemática, Pedagogia, Educação Física e geografia, matriculados regularmente nos cursos de Mestrado e Doutorado do Programa de Pós-Graduação em Educação da Universidade Estadual Paulista "Júlio de Mesquita Filho", campus de Presidente Prudente/SP.

O estudo iniciou-se a partir de uma aula expositiva e dialogada em que foi apresentado o Fluxograma para avaliação de recurso pedagógico de Manzini e Santos (2002), que estabelecem as 
seguintes etapas, a saber: entender a situação em que o recurso poderá ser utilizado, gerar ideias, escolher alternativas, representar ideias, confeccionar ou comprar o objeto (ou adaptar verificando os aspectos físicos, sociais, afetivos e cognitivos), avaliar o uso, e acompanhar o uso. Todos estes critérios visam compreender a necessidade do estudante evitando sua exposição.

Não obstante, fomos instigados à formar grupos para confecção de Recursos de TA de baixa tecnologia, que apresentassem função educativa e, ainda, apresentar estratégias para utilização do mesmo.

Posteriormente, cada participante da disciplina tornou-se colaborativamente um juiz com o intuito de analisar, segundo os princípios de Manzini e Santos (2002) e Cook e Hussey (1995) se o recurso construído poderia ser considerado um recurso de TA com finalidade pedagógica em um grupo de EPAEE. De acordo com Cook e Hussey (1995) existem oito critérios que devem ser considerados durante a análise de um recurso de TA, a saber: não exigir movimentos inapropriados durante seu uso; não despender de grande gasto energético durante seu uso; ser seguro e confortável para o usuário; ter baixo custo; ser de fácil movimentação; ser personalizado às necessidades do indivíduo; ser durável, e, ter boa aceitação social, não expondo o usuário.

Após a confecção, descrição de estratégias e apresentação do recurso. $O$ grupo de profissionais, participantes da disciplina, analisou e sugeriu novas readequações para atender os critérios de um recurso de TA de modo que foram adequadas e reavaliado o recurso.

Com o intuito de desenvolver atividades de ensino, pesquisa e extensão, e divulgar os dados obtidos, o CPIDES, vinculado ao grupo de pesquisa Ambientes Potencializadores para Inclusão (API), se submeteu ao Comitê de Ética em Pesquisa (CEP), sendo o protocolo de aprovação: 106/2009. Diante da aprovação do comitê, os autores deste artigo, participantes da disciplina supracitada e do grupo de pesquisa API, possuem autorização para divulgação dos dados.

Assim, apresentamos a seguir a análise de um recurso de TA confeccionado bem como sua utilização no contexto escolar.

\section{RESULTADOS}

A disciplina iniciou-se com discussões sobre o conceito de TA, tecnologias aplicadas à educação e recursos pedagógico. Logo em seguida, foi proposto a todos os participantes a formação de grupos para elaboração e confecção de recursos de TA de baixa tecnologia, que, pudessem ser utilizados no contexto escolar com EPAEE. Desta forma, cada grupo se reuniu para confecção dos recursos, ao todo, obtivemos cinco recursos para serem analisados no decorrer da disciplina.

Foram confeccionados os seguintes recursos: um cubo mágico tátil para estudantes com deficiência visual, um jogo de xadrez tátil para estudantes com deficiência visual, um quadro imantado sobre o sistema de numeração para estudantes com deficiência física, um quadro imantado sobre alfabetização para estudantes com deficiência física e um jogo sobre sequência lógica para estudantes com transtorno do espectro autista.

As regras para confecção dos recursos supracitados foram claras e simples, os recursos deveriam ser caracterizados como sendo de baixa tecnologia, possíveis de serem confeccionados no ambiente escolar, e que fossem de fato um recurso de TA, ou seja, que proporcionasse ao usuário uma funcionalidade.

Desta forma, confeccionamos, primeiro e segundo autor deste artigo, o cubo mágico tátil, para tanto utilizamos seis texturas distintas, segue abaixo a figura: 


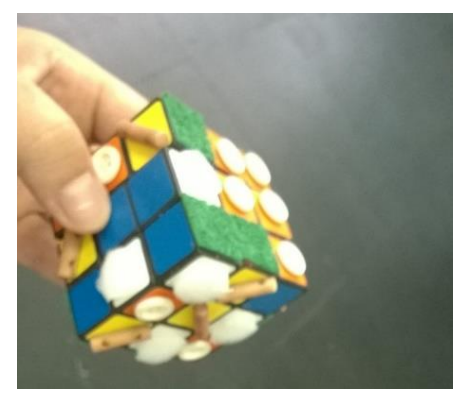

Figura 01. Primeira versão do Cubo Mágico Tátil

Fonte: Imagem disponibilizada pelos autores

Pensamos em adaptar o cubo mágico para os estudantes com deficiência visual. Ao invés de utilizarmos um cubo mágico com cores diferentes, adaptamos para o estudante cego e construímos um cubo com diversas texturas, a fim de que ele possa realizar a mesma atividade que os demais estudantes com autonomia e segurança.

O desafio começou quando cada um dos participantes se tornaram juízes, e tinham por função avaliar os recursos dos outros grupos, levando em consideração os critérios estabelecidos por Manzini e Santos (2002) e Cook (1995). Dentre eles, deveríamos avaliar se os recursos produzidos eram realmente um recurso de TA ou um simplesmente um recurso pedagógico, levando em consideração as estratégias para utilização.

Esta foi uma tarefa complexa de ser realizada, visto a dificuldade de atender a necessidade do estudante para desempenhar a tarefa com o recurso escolhido.

Com as características apresentas na Figura 1, o cubo mágico, para nós, deixaria de ser um recurso pedagógico para ser um recurso de Tecnologia Assistiva, pois para o estudante com deficiência visual, o cubo mágico adaptado pode auxiliar em termos de localização e espaço, visto que demanda determinadas habilidades para seu manuseio.

Porém, os juízes apontaram diversas mudanças a serem realizadas a fim de melhorar o recurso, como a substituição de objetos pequenos que eram fáceis de serem descolados do cubo, por tecidos e papéis com texturas. Além disso, realizaram contribuições referente às estratégias de utilização do mesmo, como para construir conceitos matemáticos, como o de vértice, aresta, face, entre outros.

Com isso, nos reunimos novamente para adaptar o cubo mágico tátil, conforme as contribuições dos juízes. Assim, o cubo mágico tátil se transformou em um cubo inteligente, de acordo com a figura abaixo:

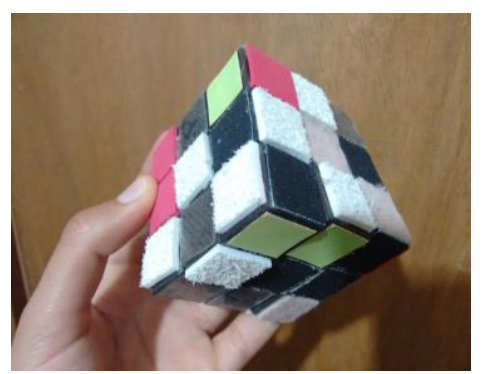

Figura 02. Versão Final do Cubo Mágico Tátil: “Cubo Inteligente Fonte: Imagem disponibilizada pelos autores

O cubo mágico tátil adaptado para pessoas com deficiência visual passou a ser chamado "Cubo Inteligente", após a avalição dos juízes percebeu-se que além de ser utilizado pelo professor da Sala de Recursos Multifuncionais, para desenvolver habilidades relacionadas à concentração, desenvolvimento de raciocínio lógico e elaboração de estratégias para montar o cubo de maneira 
correta, pode, também, ser utilizado pelo professor da sala de aula comum para trabalhar com conteúdo da geometria.

Ao manusear o cubo inteligente, o estudante poderá, com orientação do professor, construir conceitos relacionados à face, vértice e aresta dos poliedros, diferenciar figuras pela sua forma (poliedros de corpos redondos, por exemplo) e construir conceitos relacionados à Geometria, a partir da ação sobre o objeto.

Para que os estudantes desenvolvam habilidades relacionadas ao raciocínio logico, concentração e organização do pensamento (essenciais para a aprendizagem de Matemática) o Cubo Inteligente pode ser utilizado com a mesma função que é utilizado para os estudantes que não possuem deficiência visual. Durante as aulas, os estudantes tentarão juntar as partes iguais para compor as faces do cubo. Além das habilidades já mencionadas, o professor da sala de aula comum poderá também utilizar o mesmo recurso para trabalhar com as partes dos poliedros: faces, vértices e arestas. É possível também trabalhar com o cubo mágico, monitorando o tempo levado para a formação individual das faces ou de todo o quebra cabeça.

Nesse sentido, professores do Atendimento Educacional Especializado e da sala de aula comum poderão utilizar o mesmo recurso para desenvolver seu trabalho de forma colaborativa. É necessário ressaltar que o cubo também pode ser utilizado por todos os demais estudantes, garantindo uma boa aceitação do recurso por todos.

Entendemos que a TA é o conjunto de serviços e instrumentos que objetivam maximizar as potencialidades dos indivíduos com alguma deficiência, melhorar a independência funcional, aumentar a interação social e evidentemente, melhorar sua qualidade de vida e das pessoas que o cercam. Por isso, chegamos a conclusão de que seguir os passos indicados pela literatura e seus critérios para confeç̧ão de um recurso de TA é extremamente importante para aceitação do mesmo pelo EPAEE. Além disso, o trabalho colaborativo proporciona ao recurso adaptações que não faríamos sem um olhar crítico. Vale lembrar que, no âmbito escolar é importante que o EPAEE participe ativamente do processo de avaliação do recurso de TA, pois este visa oferecer funcionalidade para a vida diária, autonomia e segurança.

\section{DISCUSSÃO}

O termo Tecnologia Assistiva (TA) ainda é novo, pois emergiu no cenário brasileiro por volta da década de noventa. Segundo Garcia e Galvão Filho (2012), a TA vem se tornando, cada vez mais, uma ponte para abertura de um novo horizonte nos processos de aprendizagem, desenvolvimento, autonomia e independência de idosos e pessoas com deficiência, incluindo até aquelas consideradas bastante severas.

Podemos considerar a TA como uma conquista herdada do avanço científico e tecnológico, visto que viabilizada, por meio de pesquisas relacionadas à área da inclusão estabelece a criação de equipamentos, serviços, estratégias e práticas para melhor autonomia de Pessoas com Deficiência (PCD).

Em 2006 a Secretaria dos Direitos Humanos da Presidência da República- SEDH/PR, por meio da portaria no 142, instituiu o Comitê de Ajudas Técnicas (CAT) que define a Tecnologia Assistiva como

uma área do conhecimento, de característica interdisciplinar, que engloba produtos, recursos, metodologias, estratégias, práticas e serviços que objetivam promover a funcionalidade, relacionada à atividade $e$ participação, de pessoas com deficiência, incapacidades ou mobilidade reduzida, visando sua autonomia, independência, qualidade de vida e inclusão social. 
Conforme Brasil (2009) a classificação de TA mais utilizada é a ISO 9999. A International Organization for Standardization (Associação Internacional de Normalização) é uma federação mundial composta por associações nacional. $\mathrm{O}$ trabalho de preparar as normas internacionais é geralmente executado pelos comitês técnicos da ISO.

A classificação apresenta-se em três níveis diferentes: classe, subclasse e detalhamento da classificação, com explicações e referencias. O primeiro nível mais geral de classificação tem onze classes de produtos assistivos, respectivamente, para: Tratamento médico pessoal; Treinamento de habilidades; Órteses e próteses; Proteção e cuidados pessoais; Mobilidade pessoal; Cuidados com o lar; Mobiliário e adaptações para residenciais e outras edificações; Comunicação e informação; Manuseio de objetos e equipamentos; Melhorias ambientais, ferramentas e máquinas; Lazer.

Os recursos de tecnologia assistiva estão muito próximos do nosso dia-a-dia. Ora eles nos causam impacto devido à tecnologia que apresentam, ora passam quase despercebidos. Para exemplificar, podemos chamar de tecnologia assistiva uma bengala, utilizada por nossos avós para proporcionar conforto e segurança no momento de caminhar, bem como um aparelho de amplificação utilizado por uma pessoa com surdez moderada ou mesmo veículo adaptado para uma pessoa com deficiência. (MANZINI, 2005, p. 82)

Além disso, os recursos de TA podem ser classificados como sendo de alta, média ou baixa tecnologia. Sendo os de baixa tecnologia aqueles de custo mais acessível, podendo até mesmo serem confeccionados. Os recursos de média tecnologia são aqueles elétricos que não necessitam de um sistema computacional. Os de alta tecnologia englobam softwares especiais, adaptados e mais complexos.

A TA, enquanto serviço, tem por finalidade realizar a avaliação, prescrição e ensino da utilização de um recurso apropriado a determinado indivíduo. Este processo, deve envolver diretamente o usuário, pois deverá tomar por base o conhecimento de seu contexto, a valorização de suas intenções e necessidades funcionais pessoais, bem como suas habilidades e potencialidades atuais.

Em âmbito educacional, a TA geralmente remete a ideia de um recurso/objeto que foi planejado e construído com uma finalidade a fim de possibilitar uma funcionalidade ao indivíduo que o utilizará, e se esquece que este fato também se refere à uma prestação de serviços, envolvendo uma técnica, um procedimento, uma estratégia de aplicação.

A partir destas premissas e das discussões que aconteceram durante aulas da disciplina, compreendemos que, apesar de ainda haver a necessidade de se aprofundar nas leituras para definir o que é TA, é possível diferenciá-la da Tecnologia Educacional (TE) ou das Tecnologias Digitais da Informação e Comunicação (TDIC)

\section{CONCLUSÃO}

A partir das inúmeras análises em relação aos recursos, os participantes chegaram à conclusão de que um recurso só pode ser caracterizado como TA a partir do momento que o seu objetivo de utilização seja o de maximizar ou oportunizar ao indivíduo com alguma deficiência meios para que ele consiga realizar atividades de maneira autônoma. Os recursos de TA contribuem de maneira significativa, como estratégia, para o processo de ensino e aprendizagem dos estudantes, pois ao se divertir com situações pedagógicas mediadas pelo professor, o sujeito passa a construir seu próprio conhecimento. Entretanto, para que o estudante com deficiência seja ativo no processo de construção de conhecimento, são necessários recursos de TA para que 
ele possa, a partir de seus interesses e conhecimento, exercitar sua capacidade funcional de pensar de modo autônomo.

\section{REFERÊNCIAS}

BRASIL, Ata da Reunião III, de abril de 2007, Comitê de Ajudas Técnicas, Secretaria Especial dos Direitos Humanos da Presidência da República (CORDE/SEDH/PR), 2007.

BRASIL: Ministério da Educação. Secretaria de Educação Especial. Política Nacional de Educação Especial na perspectiva da Educação Inclusiva. Brasília: MEC/SEESP, 2007.

Brasil. Subsecretaria Nacional de Promoção dos Direitos da Pessoa com Deficiência. Comitê de Ajudas Técnicas. Tecnologia Assistiva. - Brasília: CORDE, 2009.

COOK, A.M. \& HUSSEY, S. M. (1995) Assistive Technologies: Principles and Practices. St. Louis, Missouri. Mosby - Year Book, Inc.

COOK, DB, Sackett DL, Spitzer WO. Methodologic guidelines for systematic reviews of randomized controlled trials in health care from the Potsdam consultation on meta-analysis. J Clin Epidemiol, 1995; 48: 167-171. https://doi.org/10.1016/0895-4356(94)00172-M

GARCIA, J. C. D. GALVÃO FILHO, Teófilo A. Pesquisa Nacional de Tecnologia Assistiva. São Paulo: ITS BRASIL, 2012.

MANZINI, E. J. 2005. Tecnologia assistiva para educação: recursos pedagógicos adaptados. In: Ensaios pedagógicos: construindo escolas inclusivas. Brasília: SEESP/MEC, p.82-86.

MANZINI, E. J. ; SANTOS, M. C. F. Portal de ajudas técnicas para a educação: equipamento e material pedagógico para educação, capacitação e recreação da pessoa com deficiência - recursos pedagógicos adaptados. 1. ed. Brasília: MEC, . v. 1, 2002, $56 \mathrm{p}$

RADABAUGH, M. P. NIDRR's Long Range Plan - Technology for Access and Function Research Section Two: NIDDR Research Agenda Chapter 5: TECHNOLOGY FOR ACCESS AND FUNCTION, 1993. Disponível em: http://goo.gl/RxHiyK. Acesso em: 02 abr 2016. 